\title{
PRIMERA REUNIÓN SOBRE EL REBECO CANTÁBRICO Y EL SARRIO PIRENAICO. BENASQUE (HUESCA, ESPAÑA), 20-22 DE MAYO 2011
}

\author{
J.L. Alarcón ${ }^{1}$, D. Fernández de Luco ${ }^{2}$, R. García-GonZÁlez ${ }^{3}$, \\ A. García-Serrano 4 \& J. Herrero 5 (COMité Organizador) \\ 1. Gobierno de Aragón \\ 2. Departamento de Patología. Universidad de Zaragoza. Miguel Servet 177. E-50013 Zaragoza. \\ luco@unizar.es \\ 3. Instituto Pirenaico de Ecología, CSIC. Apdo. Correos 64, 22700 Jaca (Spain), rgarciag@ipe.csic.es \\ 4. Ega Consultores en Vida Silvestre. Sierra de Vicort 31. E-50003 Zaragoza, Spain. egasl@arrakis.escon \\ 5. Área de Ecología. Escuela Politécnica Superior de Huesca. Universidad de Zaragoza. E-22071 \\ Zaragoza.herreroj@unizar.es
}

\begin{abstract}
Antecedentes y objetivos
En 2002 se celebraron las primeras jornadas sobre el sarrio pirenaico en Jaca (Huesca). Constituyó de encuentro importante para todos los gestores, científicos y cazadores interesados en la especie, que permitió compartir experiencias y establecer relaciones entre especialistas de ambas vertientes de la cadena pirenaica. Las jornadas fueron posteriormente publicadas en un volumen monográfico que aunaba el conocimiento básico sobre la biología del sarrio pirenaico (Rupicapra pyrenaica pyrenaica) con aspectos de la patología y gestión de sus poblaciones (Herrero et al. 2004). Pocos años más tarde surgió una iniciativa similar sobre el rebeco cantábrico (R.p. parva). Tras unas jornadas celebradas en Asturias en 2006, se publicó también un libro con las principales contribuciones junto con otros trabajos posteriores (Pérez-Barbería \& Palacios, 2009). Tras casi una década desde la primera iniciativa y con el fin de actualizar el estado del conocimiento de las poblaciones de rupicaprinos del sudoeste europeo, se celebraron unas nuevas jornadas en Benasque (Huesca) en mayo de 2011, que reunieron a diferentes sectores interesados en este ungulado autóctono de alto valor ecológico y económico. A continuación se transcriben los resúmenes de las comunicaciones presentadas.
\end{abstract}




\title{
LOS NOMBRES POPULARES DEL GÉNERO RUPICAPRA: REBECO Y SARRIO
}

\author{
R. VIDALLER TRICAS \\ Caldearenas 18. E-22600 Samianigo
}

Existe lo que tiene nombre, y el nombre encuadra a lo que existe en taxonomías que permiten organizar el saber, manejarlo, dentro de marcos culturales concretos. El análisis de estas culturas nos remite a formas diversas de ver y entender el entorno. Los nombres comunes del género Rupicapra, a partir del aragonés "sarrio", nos permiten descubrir formas diferentes de relacionarnos con estas especies y de replantearnos la clasificación científica, que al fin y al cabo es una forma cultural más de organizar la biodiversidad.

Así, tanto la descripción de "sarrio" como "crapa chicota" (cabra pequeña), como los nombres secundarios (crapa... para las hembras, crapitos... para las crías), nos llevan a buscar entre los nombres de las diferentes cabras y cabras pequeñas (= Capreolus) indoeuropeos, donde encontramos denominaciones como sarne, serne, sergue, surna o srne que comparten sarrios y corzos en la Europa central y oriental, o los derivados de 'cabra' en las zonas sudorientales, desde los Alpes al Caucaso (koza, keçi, ayts...). A su vez, nombres cercanos taxonómicamente al sarrio como "bucardo" y "erco" (Capra pyrenaica), nos remiten a otros europeos de la misma cabra (bucardo), o del ciervo y el alce (erco), mientras que el gamo nos recuerda a la gamuza centroeuropea.

Al fin, se dibujaría un panorama en el que el corzo se separa del ciervo para ir junto a las cabras, mientras el ciervo y el alce forman grupo aparte con reminiscencias comunes. Una visión popular previa y diferente de la científica que nos obliga a replantear, por ejemplo, lo oportuno de asignar los nombres comunes a la taxonomía linneana (un 'sarrio' lo es tanto en Cantabria como en el Caucaso), que se organiza desde otros principios, por cierto, a menudo replanteados. Por otro lado, la existencia o no de determinados nombres populares puede correlacionar con la presencia histórica de algunas especies, como en el caso del gamo.

Palabras clave: sarrio, rebeco, Rupicapra, nombre común. 
JORNADAS

\title{
REINTRODUCCIÓN DEL REBECO CANTÁBRICO EN LA MONTAÑA ORIENTAL, CANTABRIA
}

\author{
C. Sánchez Martínez, L. García Fuente, B. Serrano García \\ \& M. RODRÍGUEZ \\ Biogestión-consultora ambiental. Fundación Naturaleza y Hombre. \\ fundacion@fnyh.org.www.fnyh.org
}

Fundación Naturaleza y Hombre (FNYH), ONG ambiental, estudió y realizó la reintroducción de rebeco cantábrico Rupicapra pyrenaica parva en la Montaña Oriental de Cantabria, de donde se extinguió hace más de 150 años. Este proyecto se enmarcó en el LIFE-Naturaleza Recuperación y conservación de la biodiversidad en la cuenca del río Asón (2002-2005), promovido por FNYH con la financiación de la Comisión Europea. La Montaña Oriental de Cantabria se ubica en el sector más oriental de la Cordillera Cantábrica, incluye las cuencas fluviales de los ríos Pas, Miera y Asón y Castro Valnera (1718 $\mathrm{m}$ ) es la cumbre más elevada. El proyecto se concentró en los términos municipales de San Roque de Riomiera, Soba, Ruesga y Arredondo, donde se liberaron los rebecos y donde posteriormente se han asentado los grupos de población.

La desaparición del rebeco del área del Alto Asón comenzó en el siglo XVIII debido a la devastación de su hábitat, provocada por la tala masiva de árboles destinados a la industria naval y a la presión cinegética. La especie no consiguió adaptarse, lo que provocó su acantonamiento en el área de Picos de Europa. Los últimos rebecos de los que se tiene testimonio en la Montaña Oriental databan de 1845 en Lanchares y San Pedro del Romeral. En la actualidad, las presiones al ecosistema que ocupaba el rebeco han disminuido y ha sido posible realizar su reintroducción. Entre 2002 y 2003 se liberaron un total de 26 rebecos procedentes de la zona cántabra del Macizo Central o de los Urrieles, en los Puertos de Salvorón, el Monte Quebres y los Puertos de Áliva. Cada ejemplar se liberaba con un collar radiotransmisor que permitió el seguimiento del proceso de adaptación de los rebecos a su nuevo hábitat. En la actualidad, la población asciende a 95 rebecos, con 19 nuevas crías en 2010.

Palabras clave: reintroducción, rebeco cantábrico, Montaña Oriental, Cantabria, ONG. 


\title{
ASPECTOS ECONÓMICOS DE LA GESTIÓN DE LAS RESERVAS DE CAZA DE SARRIO
}

\author{
M. J. DOMÍNGUEZ \& A. PARís
}

Aneto Promoción y Comunicación SL, Zaragoza.www.aneto.biz, aneto@aneto.biz

Las Reservas de Caza son espacios para la promoción, fomento y protección de determinadas especies cinegéticas. En el Pirineo aragonés existen cuatro reservas para el sarrio, creadas en 1966 por sus "excepcionales posibilidades cinegéticas" con el objeto de "garantizar la difícil pervivencia de especies". En conjunto abarcan 131.804 has y la titularidad cinegética corresponde por ley al Gobierno de Aragón. El departamento competente en medio ambiente gestiona estos terrenos cinegéticos de forma directa velando por la conservación de las especies y "subordinando a esta finalidad el aprovechamiento cinegético".

Los costes de la gestión se estiman en promedio en 1.042.369,68 € / año $(7,9$ $€ /$ ha) para el periodo 2004-2010, desglosados en los siguientes conceptos: $38 \%$ personal, $33 \%$ funcionamiento, $23 \%$ subvenciones a los ayuntamientos y $6 \%$ asistencias técnicas. La evolución del coste anual estimado es relativamente estable. En los municipios, la repercusión socioeconómica directa alcanzaría tres cuartas partes de los costes de gestión, correspondientes al personal, las subvenciones y al menos la mitad de las partidas de funcionamiento. A estas aportaciones directas hay que sumar los ingresos obtenidos de las subastas de los permisos asignados a los propietarios y la cuota complementaria por pieza cazada. Además, el turismo asociado a la caza origina otras aportaciones económicas directas e indirectas. La caza en las Reservas de Caza origina unos ingresos a la Administración gestora por permisos concedidos. Según el tipo de cazador se distribuyen de la siguiente manera: $62 \%$ de cazadores nacionales, $20 \%$ de los permisos de los propietarios y $18 \%$ de cazadores locales. El ratio ingresos/costes sólo alcanza un promedio del 7\%. La gestión prioriza la conservación de recursos.

Palabras clave: economía, inversión, costes, ingresos, tarifas, rendimiento. 
JORNADAS

\title{
TRAJECTOMETRIE DES CHAMOIS: COMMENT BOUGERAIS-JE SI J'ETAIS UN CHAMOIS?
}

\author{
Z. TABLADO ${ }^{1}$, A. LOISON ${ }^{1}$, Bourgoing ${ }^{2}$, G. GarelL ${ }^{3} \&$ DubraY, D. ${ }^{4}$ \\ ${ }^{1}$ CNRS UMR 5553 - Laboratoire d'Ecologie Alpine -Le Bourget du Lac 73376 \\ ${ }^{2}$ VetAgro Sup - Campus vétérinaire de Lyon-CNRS UMR 5558 Marcy l'Etoile 69280 \\ ${ }^{3}$ Office National de la Chasse et de la Faune Sauvage- CNERA FM - 147 route de Lodève - Juvignac \\ 34990 \\ ${ }^{4}$ Office National de la Chasse et de la Faune Sauvage, 95 rue Pierre Flourens - Montpellier 34098
}

Les chamois utilisent leurs capacités de déplacement pour interagir avec leur environnent. Ces mouvements dépendent à la fois des caractéristiques et motivations propres de l'individu, comme l'âge, le patron d'activité ou le statut de reproduction, et des caractéristiques environnementales, telles que la disponibilité de ressources alimentaires ou de refuge, et le climat. En identifiant quels sont les facteurs qui affectent les déplacements des chamois, on pourra comprendre comment ils répondront à des altérations futures de leur habitat et du climat.

Nous avons ainsi étudié les trajectoires de chamois à partir de localisations de 30 femelles équipes avec des colliers GPS dans la Reserve National de Chasse et Faune Sauvage des Bauges (France). Nous avons examiné le taux de déplacements des individus à différentes échelles temporelles en regardant les associations entre mouvement, activité et caractéristiques intrinsèques et externes. Nous nous sommes intéressés plus spécifiquement aux effets du climat (température, hauteur de neige) et du dérangement par le tourisme et nous discutons aussi les possibles implications que le futur régime climatique pourra avoir sur les patrons de mouvement et d'activité de cette espèce.

Mots clés: chamois, Rupicapra rupicapra, déplacements, localisations GPS, climat, activité. 
JORNADAS

\title{
LES INDICATEURS DE CHANGEMENT ECOLOGIQUE POUR LA GESTION DES POPULATIONS D'ISARDS
}

\author{
D. Maillard ${ }^{1}$, M. Garel ${ }^{1}$, A. Loison ${ }^{3}$, D. DubraY ${ }^{1}$, J. ApPolinaire, ${ }^{1}$ \\ \& G. BOURGOIN ${ }^{2}$ \\ ${ }^{1}$ Office National de la Chasse et de la Faune Sauvage - CNERA FM - 147 route de Lodève - Juvignac \\ 34990, France \\ 2 École Nationale Vétérinaire de Lyon - CNRS UMR 5558 Marcy l'Etoile 69280, France \\ ${ }^{3}$ CNRS UMR 5553 - Laboratoire d'Écologie Alpine - Le Bourget du Lac 73376, France
}

Grâce aux marquages d'un grand nombre de chamois et d'isards réalisés depuis plus de 20 ans sur nos territoires d'étude, nous avons montré que les comptages traditionnels sous-estimaient, parfois fortement, l'effectif réel des populations suivies. Ces résultats remettent en cause l'utilisation d'estimations d'effectifs basées uniquement sur des dénombrements pour définir des plans de gestion. Nous proposons plutôt aujourd'hui de baser ces plans de gestion sur le suivi à long terme d'un faisceau d'indicateurs caractérisant la relation population-environnement: indicateurs d'abondance, indicateurs de condition et indicateurs de consommation.

Dans ce but, nous avons mis en évidence chez le chamois et l'isard que l'observation répétée d'animaux le long d'itinéraire pédestre pré-déterminé pouvait être utilisée comme un indicateur des variations d'abondance de la population suivie (Loison et al., 2006, Dubray et Groupe ICE, 2008). Le poids des jeunes de l'année est quant à lui un indicateur sensible aux conditions environnementale et à la densité d'animaux (Garel et al., sous presse) et peut donc à ce titre servir d'indicateur de performance pour le suivi des populations. Enfin l'indice de reproduction (nombre de jeunes observés par femelle adulte) pourrait jouer le même rôle que le suivi du poids des jeunes dans la mesure où chez les ongulés la performance reproductive est étroitement liée aux conditions environnementales et aux variations de densité. La pertinence de cet indicateur comme mesure de la performance reproductive réelle de la population est à l'heure actuelle en test dans un de nos territoires de référence pour le suivi de l'isard.

Cette approche basée sur le suivi simultanée de plusieurs indicateurs est dépendante de l'application rigoureuse des protocoles si l'on veut pouvoir réaliser une gestion efficace et durable des populations d'isards et de chamois.

Mots clés: isard, Rupicapra pyrenaica, ICE, IPS, poids, gestion des populations. 


\title{
NITRÓGENO FECAL, PARÁSITOS Y ESTATUS NUTRICIONAL EN EL REBECO
}

\author{
D. Gassó ${ }^{1}$, J. Bartolomé2 ${ }^{2}$ D. Ferrer ${ }^{3}$, G. Mentaberre ${ }^{1}$, G. Gálvez-Ceróna ${ }^{2}$, \\ N. NAVARRO-GONZÁLEZ ${ }^{1}$, L. FERNÁNDEZ-SIRERA ${ }^{1}$, J.R. LÓPEZ-OLVERA ${ }^{1}$, \\ X. FERnÁNDEZ-Aguilar ${ }^{1}$, L. ROSSI ${ }^{4}$, S. LAVÍN ${ }^{1}$, I. MARCO ${ }^{1} \&$ E. SERRANO ${ }^{1}$ \\ ${ }^{1}$ Servei d' Ecopatología de Fauna Salvatge (SEFaS), Departament de Medicina i Cirurgia Animals, \\ Universitat Autònoma de Barcelona (UAB), E-08193, Bellaterra, Barcelona, Spain. * emmanuel.serra- \\ no@uab.cat \\ ${ }^{2}$ Departament de Ciència Animal i dels Aliments, Universitat Autònoma de Barcelona (UAB), E-08193, \\ Bellaterra Barcelona, España. \\ ${ }^{3}$ Laboratori de Parasitologia Veterinaria, Departament de Sanitat i d'Anatomia Animals, Bellaterra, \\ Barcelona, España. \\ ${ }^{4}$ Dipartimento de Produzioni Animali, Epidemiologia ed Ecologia, Universtità di Torino, Italia.
}

De forma general, los nematodos gastrointestinales (NGI) influyen sobre la condición nutricional de los rumiantes, reduciendo el apetito (anorexia) o alterando la capacidad de absorción de nutrientes. Este efecto negativo de los NGI, ha motivado el desarrollo de varias investigaciones para averiguar qué estrategias alimentarias maximizan la cantidad de energía ingerida con un mínimo riesgo de depredación o parasitismo ("Foraging theory"). Los rebecos viven en ecosistemas donde la disponibilidad de alimento sufre fuertes variaciones estacionales. Según esta teoría, en verano los rebecos deberían decidir si alimentarse en los pastos más nutritivos, pero quizás muy utilizados y probablemente con muchos NGI, o comer en zonas de peor calidad pero más seguras. La determinación del nitrógeno fecal (Nf) es la técnica más habitual para evaluar la calidad nutricional de las poblaciones de ungulados salvajes, ya que existe una relación directa entre la calidad del forraje y el porcentaje de nitrógeno en las heces. Pero los NGI interfieren en la absorción de nutrientes, y una mayor intensidad de parasitación puede también significar una mayor pérdida de proteínas y un aumento de Nf. Durante mayo de 2009 a septiembre de 2010 estudiamos los patrones estacionales de cargas de NGI y porcentaje de $\mathrm{Nf}$ en heces de rebeco (Rupicapra pyrenaica) y de oveja en la RNC de Freser-Setcases (Pirineo Catalán). En contradicción con la "Foraging theroy", las mayores cargas parasitarias de rebecos y ovejas coincidieron con las mejores calidades nutricionales (mayor Nf). Posiblemente los animales prefirieron comer mejor aunque con más parásitos, o el Nf aumentó debido a una peor absorción del alimento. En esta comunicación, discutiremos los patrones estacionales de NGI y Nf de rebecos y ovejas y presentaremos un diseño experimental para aclarar los usos y limitaciones del Nf para monitorizar el efecto de los parásitos en poblaciones de ungulados salvajes.

Palabras clave: carga parasitaria, "Foraging theory", indicadores fecales. 


\title{
AZAPERONA Y MORTALIDAD DE REBECOS DURANTE CAPTURAS CON RED VERTICAL
}

\author{
G. Mentaberre, E. Serrano, J.R. López-Olvera, E. Casas-Díaz, R. Velarde, \\ I. MARCO \& S. LAVÍN
}

Servei d'Ecopatología de la Fauna Salvatge (SEFaS), Facultat de Veterinària, Universitat Autònoma de Barcelona, 08193, Bellaterra, Barcelona

La captura de animales salvajes puede tener distintas motivaciones. Independientemente de éstas, es necesario tener en cuenta el bienestar animal y minimizar el estrés de la captura. El uso de tranquilizantes puede ayudar a este fin, si bien se deben realizar estudios para determinar los principios activos y dosis más adecuados para cada especie y método de captura.

En esta comunicación, presentamos el análisis de las causas que determinaron varios episodios de mortalidad durante operaciones de captura física con red vertical de rebecos pirenaicos (Rupicapra pyrenaica pyrenaica) destinadas a evaluar la acción de distintos tranquilizantes. Los parámetros clínicos de los rebecos que murieron se compararon con los del resto de animales mediante análisis de la varianza. Además, utilizamos una selección de modelos estadísticos mediante el criterio de información de Akaike para evaluar la influencia del tipo de tranquilizante (acepromazina, haloperidol y azaperona), la dosis utilizada, el número de operarios por animal y la zona.

La concentración sérica de lactato -indicador de fatiga- fue significativamente superior en los rebecos que murieron y los dos modelos seleccionados indican que las dosis altas de azaperona $y$, en menor medida, de haloperidol aumentan el riesgo de muerte. Estos resultados, junto a las alteraciones observadas en las necropsias, sugieren que los animales que llegaron más fatigados a la red de captura fueron más sensibles a los tranquilizantes y experimentaron efectos secundarios adversos que incrementaron su probabilidad de morir. Esto nos lleva a pensar que las butirofenonas, especialmente la azaperona, tienen un margen de seguridad estrecho en el rebeco, contrariamente a lo descrito para otras especies de ungulados salvajes. Dada la ausencia de bibliografía, alertamos de que esta sensibilidad a las butirofenonas también podría darse en otras especies de caprinos.

Palabras clave: acepromazina, azaperona, captura física, haloperidol, lactato, mortalidad, rebeco, Rupicapra pyrenaica. 
JORNADAS

\title{
IDENTIFICACIÓN DE INDICADORES DE CAMBIO CLIMÁTICO EN UNA POBLACIÓN DE ISARD (RUPICAPRA P. PYRENAICA) EN ANDORRA
}

\author{
L. RIBA MANDICÓ \\ Àrea d'Hàbitats i Fauna, Departament d'Agricultura i Patrimoni Natural, Govern \\ d'Andorra. landry_riba@govern.ad
}

La población de isard de la reserva de caza de Enclar (Andorra) ha sido gestionada desde su creación apoyando las decisiones de gestión en los resultados de los censos simultáneos. Para poder trabajar con más parámetros y mejorar el nivel de conocimiento de la dinámica de la población, se planteó durante el año 2006 analizar los datos biométricos, parasitológicos y sanitarios, obtenidos en todos los animales cazados desde 2001. El objetivo fue identificar indicadores que permitieran "observar" los cambios en la población, inducidos por diferentes parámetros ambientales. En este trabajo se presentan los resultados preliminares del análisis realizado a partir de los animales cazados entre el 2001 y 2010, así como, las tendencias observadas en algunas de las variables analizadas en relación con parámetros de tipo ambiental o demográfico. Estos resultados preliminares permiten identificar qué parámetros podrían ser los mejores indicadores para la población estudiada, destacando como posibles variables válidas, el peso de la canal y las medidas asociadas con los cuernos (longitud, altura y perímetro de la base), siendo a la vez las menos sujetas a un "efecto manipulador" en relación a otras variables morfológicas. La tendencia mostrada por estas variables en los animales que se encuentran en crecimiento (en teoría, más sensibles a los cambios ambientales), indica una disminución progresiva de los valores en el tiempo, mostrando además una correlación negativa con la densidad estimada de la población, durante los años anteriores al muestreo, así como con algunas variables climatológicas analizadas. Otros datos de tipo clínico o fisiológico (valores de excreción parasitaria, morfología de las glándulas adrenales) se encuentran actualmente en estudio.

Palabras claves: isard, Andorra, Enclar, indicadores. 


\title{
VARIACIONES ESTACIONALES DE NITRÓGENO FECAL EN EL REBECO MEDIANTE NIRS
}

\author{
A. GÁlvez-Cerón ${ }^{1}$, E. Albanell ${ }^{1}$, E. Serrano ${ }^{2}$, G. Mentaberre ${ }^{2}$, X. \\ FERnÁNDEZ-AGUILAR ${ }^{2}$, L. FERnÁNDEZ ${ }^{2}$, N. NAVARRO-GONZÁlEZ2 ${ }^{2}$, J.R. LÓPEZ- \\ OLVERA ${ }^{2}$, S. LAVÍN ${ }^{4}$, I. MARCO ${ }^{4} \&$ J. BARTOLOMÉ \\ ${ }^{1}$ Departament de Ciència Animal i dels Aliments, Universitat Autònoma de Barcelona (UAB), E-08193, \\ Bellaterra,Barcelona, Spain.ArturoLeonel.Galvez@uab.cat \\ ${ }^{2}$ Servei d' Ecopatología de Fauna Salvatge (SEFaS), Departament de Medicina i Cirurgia Animals, \\ Universitat Autònoma de Barcelona (UAB), E-08193, Bellaterra, Barcelona, Spain.
}

Para maximizar su aptitud biológica, los rebecos (Rupicapra pyrenaica) incrementan su condición física cuando la productividad primaria es máxima. El nitrógeno fecal (Nf) es un excelente indicador de la calidad de la dieta de los herbívoros. Monitorizar las variaciones estacionales de Nf, puede ser útil para comprender la ecología de esta especie y establecer planes de gestión adaptados a las condiciones locales. En esta comunicación, presentamos los patrones anuales de $\mathrm{Nf}$ de rebeco pirenaico a partir de 192 muestras de heces recolectadas desde mayo de 2009 hasta septiembre de 2010 en la RNC de Freser-Setcases (Pirineo Catalán). Las muestras se analizaron por el método tradicional DUMAS/LECO y se calibraron posteriormente mediante espectroscopía en el infrarrojo cercano (NIRS) por regresión MPLS (mínimos cuadrados parciales modificados). El NIRS ofrece ciertas ventajas adicionales respecto al DUMAS/LECO, ya que se puede reutilizar la muestra y no produce residuos. La dieta de los rebecos mostró un marcado patrón estacional, siendo el Nf máximo entre abril-agosto y mínimo entre septiembre-marzo. Tras observar este patrón, decidimos hacer dos calibraciones con el NIRS: [1] incluyendo muestras de todo el año y [2] dividendo el año en dos periodos [abril-agosto ( $\mathrm{Nf}$ alto) y septiembre-marzo (Nf bajo)]. Aunque los resultados para la calibración general [coeficiente determinación $\left(R^{2}\right)$ 0,97; error estándar de calibración (SEC) 0,076; coeficiente determinación de validación $\left(\mathrm{r}^{2}\right)$ 0,95 y error estándar de predicción (SEP) 0,109], garantizan una predicción adecuada del Nf, las calibraciones parciales de los dos períodos del año (Nf alto y Nf bajo) mejoraron algunos parámetros estadísticos, especialmente el SEC y SEP. Los resultados obtenidos indican que la tecnología NIRS puede ser de gran utilidad para evaluar con suficiente precisión el nitrógeno fecal en rebecos.

Palabras clave: calidad nutricional, heces, infrarrojo cercano. 
JORNADAS

\title{
REBECOS Y PARÁSITOS, UN ESBOZO MEDIANTE SISTEMAS DE ECUACIONES ESTRUCTURALES
}

\author{
E. SERRANO ${ }^{1}$, D. FerReR ${ }^{2}$, G. MENTABERRE ${ }^{1}$, N. NAVARRO-GONZÁLEZ ${ }^{1}$, L.

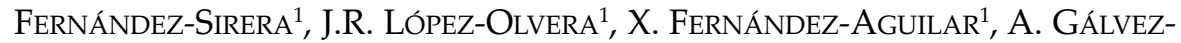 \\ CERon ${ }^{3}$, J. Bartolomé ${ }^{3}$, L. Rossi ${ }^{4}$, J. Pérez ${ }^{5}$, S. Lavín ${ }^{1} \&$ I. Marco $^{1}$ \\ ${ }^{1}$ Servei d' Ecopatologia de Fauna Salvatge (SEFaS), Departament de Medicina i Cirurgia Animals, \\ Universitat Autònoma de Barcelona (UAB), E-08193, Bellaterra, Barcelona, Spain. *emmanuel.serra- \\ no@uab.cat \\ ${ }^{2}$ Laboratori de Parasitologia Veterinaria, Departament de Sanitat i d'Anatomia Animals, Bellaterra, \\ Barcelona, Spain. \\ ${ }^{3}$ Departament de Ciència Animal i dels Aliments, Universitat Autònoma de Barcelona (UAB), E-08193, \\ Bellaterra, Barcelona, Spain. \\ ${ }^{4}$ Dipartimento de Produzioni Animali, Epidemiologia ed Ecologia, Universtità di Torino, Italy. \\ ${ }^{5}$ Departamento de Biología Animal, Biología Vegetal y Ecología, Universidad de Jaén, E- 23071, Jaén, \\ Spain.
}

En la fauna salvaje los patrones de infección no son homogéneos, ni en el tiempo ni en el espacio. Este hecho se debe principalmente a tres mecanismos: [1] hay una gran diversidad de factores de riesgo ligados a la infección (p. e., ambientales, poblacionales y de tipo humano), [2] todos los individuos de la población no tienen la misma probabilidad de entrar en contacto con el agente patógeno o, [3] no manifiestan la misma susceptibilidad a ser infectados. La importancia relativa de estos mecanismos no está esclarecida para la mayoría de la enfermedades de la fauna salvaje. En esta comunicación presentamos los path-pls, una herramienta estadística emergente para explorar relaciones causa-efecto entre factores espaciales (altitud y localidad), de la fenología local (mes del año, calidad nutricional) y poblacionales (tamaño de grupo y densidad), sobre las cargas de coccidios y nematodos gastrointestinales, en dos poblaciones de rebeco pirenaico (Rupicapra pyrenaica) de la Reserva Nacional de Caza de Freser-Setcases (Pirineo catalán). Entre otras ventajas, respecto a las herramientas estadísticas convencionales, los path-pls ponen de manifiesto relaciones indirectas entre variables que estén fuertemente relacionadas entre sí (colinealidad).

Palabras clave: modelos path-pls, relación parasito-hospedador, Rupicapra pyrenaica. 


\title{
VARIATIONS DEMOGRAPHIQUES CHEZ L'ISARD (RUPICAPRA P. PYRENAICA) D'APRES UN SUIVI PAR RECENSEMENTS HIVERNAUX STANDARDISES
}

\begin{abstract}
J. P. CRAMPE
Parc National des Pyrénées, 2 rue du IV septembre 65000 Tarbes, France. j-paul.crampe@wanadoo.fr

Un suivi hivernal par recensements répétés a été appliqué à la population d'isard protégée de Cauterets, dans le Parc National des Pyrénées. De 1992 à 2010, 290 recensements standardisés ont été réalisés de décembre à avril sur une zone d'hivernage appelée "Clot-Cayan ». La stabilité spatiale des isards sur cette zone d'hivernage a été démontrée par un suivi par marquage sur plusieurs années. Les données basées sur le comptage des effectifs des différentes classes d'âge et sexe ont permis de mettre en évidence des variations relativement importantes de certains paramètres démographiques. Parmi les facteurs invoqués, certains paraissent relever de la densité, tandis que d'autres sont de nature stochastiques et dépendent plutôt du climat hivernal ou d'évènements passagers. Ces facteurs de nature différente paraissent agir en synergie. Le passage d'une épizootie de kérato-conjonctivite de 2007 à 2008 a permis de constater un impact relativement important de cette maladie sur la taille de la population.
\end{abstract}


JORNADAS

\title{
CALENTAMIENTO GLOBAL Y HELMINTOS DEL REBECO: UNA APROXIMACIÓN EXPERIMENTAL
}

\author{
A. GÁlvez-Cerón ${ }^{1}$, J. Bartolomé ${ }^{1}$, D. Ferrer ${ }^{2}$, L. Rossi ${ }^{3}$, G. Mentaberre ${ }^{4}$, S. \\ LAVÍN $^{4}$, I. MARCO ${ }^{4} \&$ E. SERRANO ${ }^{4}$ \\ ${ }^{1}$ Departament de Ciència Animal i dels Aliments, Universitat Autònoma de Barcelona (UAB), E-08193, \\ Bellaterra, Barcelona, Spain. *ArturoLeonel.Galvez@uab.cat \\ ${ }^{2}$ Laboratori de Parasitologia Veterinaria, Departament de Sanitat i d'Anatomia Animals, Bellaterra, \\ Barcelona, Spain. \\ ${ }^{3}$ Dipartimento de Produzioni Animali, Epidemiologia ed Ecologia, Universtità di Torino, Italy. \\ ${ }^{4}$ Servei d' Ecopatologia de Fauna Salvatge (SEFaS), Departament de Medicina i Cirurgia Animals, \\ Universitat Autònoma de Barcelona (UAB), E-08193, Bellaterra, Barcelona, Spain.
}

El calentamiento global está alterando la ecología de muchas especies de parásitos de ungulados domésticos y salvajes de todo el mundo. En las últimas décadas, leves incrementos en la temperatura media anual han sido suficientes para explicar el aumento de helmintiasis en el Reino Unido o la aparición de nuevas especies de nematodos en ungulados salvajes del Ártico. De hecho, incrementos de $1,5^{\circ} \mathrm{C}\left(\mathrm{t}^{\mathrm{o}}\right.$ media diaria) son suficientes para acelerar el desarrollo de parásitos de ciclo directo o favorecer la dispersión de hospedadores intermediarios. No obstante, los efectos del calentamiento global sobre la dinámica parásito-hospedador no parecen sencillos. Un aumento de la temperatura también puede desecar las fases infectivas de los parásitos, o mejorar el estado nutricional de los hospedadores (p.ej., aumentando la productividad primaria). Esta compleja interacción entre el calentamiento global y la dinámica parásito-hospedador es difícil de abordar mediante la observación de patrones naturales, pero se puede estudiar con la ayuda de una manipulación en condiciones naturales. En esta comunicación, revisaremos los efectos del calentamiento global sobre la dinámica y distribución de los parásitos de varias especies de ungulados domésticos y salvajes, y presentaremos el diseño de una simulación de calentamiento global en la Reserva Nacional de Caza de Freser-Setcases (Pirineo Catalán). Con este experimento pretendemos estudiar la influencia de un aumento de temperatura sobre: [1] la estructura y composición de los pastos y [2] el desarrollo y supervivencia de los nematodos gastrointestinales del rebeco pirenaico (Rupicapra p. pyrenaica) y de otros ungulados domésticos (ovejas, vacas y caballos).

Palabras clave: nematodos gastrointestinales, ganadería de montaña, Rupicapra pyrenaica. 
JORNADAS

FACTORES DE RIESGO ASOCIADOS A LA PESTIVIROSIS DEL REBECO PIRENAICO

\author{
I. MARCO ${ }^{1}$, O. CABEZÓN ${ }^{1,2}$, L. FERNÁNDEZ-SiRERA ${ }^{1,2}$, J.R. LÓPEZ-Olvera ${ }^{1}$, \\ G. Mentaberre ${ }^{1}$, E. Casas ${ }^{1}$, E. Serrano ${ }^{1}$, R. Velarde ${ }^{1}$, R. Rosell ${ }^{2,3} \&$ S. LAVín ${ }^{1}$ \\ ${ }^{1}$ Servei d'Ecopatologia de Fauna Salvatge, Facultat de Veterinària, Universitat Autònoma de Barcelona, \\ E-08193, Bellaterra, Barcelona, Spain. * Ignasi.Marco@uab.cat \\ ${ }^{2}$ Centre de Recerca en Sanitat Animal (CRESA), Universitat Autònoma de Barcelona, E-08193, \\ Bellaterra, Barcelona, Spain \\ ${ }^{3}$ Departament d'Agricultura, Ramaderia, Pesca, Alimentació i Medi Natural, Generalitat de Catalunya, \\ Gran Via de les Corts Catalanes 612-614, E-08007, Barcelona, Spain
}

En 2001 un Virus de la Enfermedad de la Frontera (Género Pestivirus, Familia Flaviviridae) se asoció con una nueva enfermedad en el rebeco pirenaico (Rupicapra pyrenaca pyrenaica). Desde entonces se han sucedido numerosos brotes de enfermedad en una gran parte del Pirineo central y oriental de España, Francia y Andorra, con graves consecuencias demográficas. Los factores observados, que afectan a la aparición y gravedad de la enfermedad, son los relacionados con el propio virus, la aparición de infecciones secundarias, la inmunidad poblacional, la ecología y el comportamiento del rebeco y la variabilidad genética. Otros factores que también pueden tener influencia son las cepas del virus y los individuos persistentemente infectados. En conclusión, la Enfermedad de la Frontera del rebeco pirenaico es una enfermedad emergente que representa la principal amenaza para las poblaciones de esta especie. A pesar de que es una enfermedad difícilmente controlable, el análisis de los factores de riesgo puede ayudar a predecir la aparición de nuevos brotes de la enfermedad.

Palabras clave: Ecologia de las enfermedades, Epidemiologia, Pestivirus, Rupicapra pyrenaica. 


\title{
EFECTO DE LAS ENFERMEDADES SOBRE LA DINÁMICA POBLACIONAL DEL REBECO
}

\author{
J.R. López-OlveraA ${ }^{1}$, I. Marco ${ }^{1}$, O. Cabezón ${ }^{1,2}$, N. Navarro-GonZÁleZZ, \\ X. FernándeZ-Aguilar ${ }^{1}$, L. FernándeZ-Sirera ${ }^{1,2}$, G. MEnTABerre ${ }^{1}$, E. CASAS ${ }^{1}$, \\ R. VELARDE ${ }^{1}$, S. LAVÍN ${ }^{1} \&$ E. SERRANO ${ }^{1}$
${ }^{1}$ Servei d' Ecopatología de Fauna Salvatge, Departament de Medicina i Cirurgia Animals, Universitat Autònoma de Barcelona, E-08193, Bellaterra, Barcelona, Spain. *emmanuel.serrano@uab.cat
${ }^{2}$ Centre de Recerca en Sanitat Animal (CRESA), Universitat Autònoma de Barcelona, E-08193, Bellaterra, Barcelona, Spain

La evolución del tamaño y la estructura de las poblaciones constituyen uno de los aspectos centrales de la ecología. En los ungulados se observa que los factores estocásticos y dependientes de la densidad afectan principalmente a la supervivencia de los juveniles (reclutamiento), ya que la supervivencia de los adultos es prácticamente constante y la productividad de las hembras es poco variable entre años. Aunque se han descrito numerosas enfermedades en los ungulados salvajes, su efecto sobre las poblaciones es bastante desconocido, ya que no se ha valorado su efecto sobre cada una de las fases del ciclo vital: nacimiento, mortalidad pre-destete (supervivencia estival), mortalidad post-destete (supervivencia invernal), supervivencia entre uno y dos años, supervivencia adulta y supervivencia en la senescencia, así como la productividad de las hembras en cada una de las cuatro fases de su vida reproductora (primala, joven, adulta y senescente). En la presente comunicación abordamos una revisión de enfermedades descritas (o que podrían afectar) a la eficacia biológica y la dinámica poblacional de las especies del género Rupicapra.

Palabras clave: Ecología de las enfermedades, dinámica poblacional. 


\title{
INFLUENCE DU PESTIVIRUS SUR LA DYNAMIQUE DES POPULATIONS D'ISARDS PYRENEENS
}

\author{
M. Pioz ${ }^{1}$, E. Gilot-Fromont ${ }^{2}$, A. LOISON ${ }^{3}$, D. Dubray ${ }^{4} \&$ V. Grosbois ${ }^{5}$ \\ ${ }^{1}$ Institut National pour la Recherche Agronomique- Unité d'Epidémiologie Animale - Centre de \\ Clermont-Ferrand Theix - St Genès Champanelle 63122 maryline.pioz@hotmail.fr \\ ${ }^{2}$ Ecole Nationale Vétérinaire de Lyon - CNRS UMR 5558 Marcy l'Etoile 69280 e.gilot@vetagro-sup.fr \\ ${ }^{3}$ CNRS UMR 5553 - Laboratoire d'Ecologie Alpine-Le Bourget du Lac 73376 anne.loison@univ- \\ savoie.fr \\ ${ }^{4}$ Office National de la Chasse et de la Faune Sauvage- CNERA FM - 147 route de Lodève - Juvignac \\ 34990 dominique.dubray@oncfs.gouv.fr \\ ${ }^{5}$ CIRAD - UR AGIRs - Campus International de Baillarguet - Montpellier cedex 534398 \\ vladimir.grosbois@cirad.fr
}

Depuis l'année 2001, des épisodes de mortalité massive associés à la découverte d'isards (Rupicapra pyrenaica) malades ont été rapportés dans les Pyrénées en Espagne, en Andorre et en France. Les investigations réalisées ont permis l'isolement chez une partie des individus malades d'un Pestivirus identifié comme un Border Disease Virus (BDV). Ce virus, soupçonné d'être responsable des symptômes et mortalités observés, fut classé dans le sousgroupe Border Disease Virus-4 (BDV-4).

Des études sérologiques rétrospectives ont montré que le virus circulait depuis au moins 1995 dans la population d'isards de la Réserve Nationale de Chasse et de Faune Sauvage d'Orlu alors qu'aucune mortalité anormale n'avait été rapportée. Depuis, $90 \%$ des animaux de plus de 2 ans ont eu un contact avec le virus et la présence, chaque année, d'animaux virémiques suggère que le virus est devenu endémique dans cette population. Nous avons cherché à comprendre comment ce virus influençait la dynamique de la population d'isards en étudiant l'évolution de la survie et de la fécondité de cette population.

Motsclés: Rupicapra pyrenaica, Border Disease Virus, infection, survie, fécondité. 
JORNADAS

\title{
DIEZ AÑOS DE VIGILANCIA SANITARIA, ACTIVA Y PASIVA, EN EL SARRIO DEL PIRINEO ARAGONÉS*
}

\author{
M.C. ArnaL ${ }^{1}$, M. Revilla ${ }^{1}$, D. Martínez ${ }^{1}$, M.J. De Miguel ${ }^{2}$, P.M. Muñoz ${ }^{2}$ \\ \& D. FERNÁNDEZ DE LUCO ${ }^{1}$ \\ ${ }^{1}$ Centro de Investigación en encefalopatías y enfermedades trasmisibles emergentes. Departamento de \\ Patología Animal. Facultad de Veterinaria. Universidad de Zaragoza. C/ Miguel Servet 177, E-50013 \\ Zaragoza, España \\ ${ }^{2}$ Centro de Investigación y Tecnología Agroalimentaria (CITA). Gobierno de Aragón. \\ ${ }^{*}$ Estudio financiado por el Dpto. de Medio Ambiente y Dpto. de Agricultura y Alimentación. Gobierno \\ de Aragón.
}

La red de vigilancia sanitaria del sarrio en el Pirineo aragonés permite conocer la situación de procesos epidémicos, como pestivirosis, sarna y queratoconjuntivitis infecciosa $(\mathrm{QCI})$; zoonosis como brucelosis, tuberculosis e hidatidosis; y otros procesos patológicos en la especie. En un periodo de 10 años (2000-09) el número de sarrios enfermos-muertos analizados ha sido de 187, además de 117 animales cazados y 1.852 muestras de sarrios cazados $(\mathrm{n}=2.156)$. El seguimiento sobre pestivirosis se ha realizado en 1.459 sarrios, no detectándose casos clínicos ni sarrios infectados. El estudio serológico frente a pestivirus muestra una mayor proporción de animales seropositivos en las RC de Benasque (18,5\%) que en el resto de reservas más occidentales $(2,3 \%)$. Un brote de QCI afectó a sarrios de Aragón y Navarra desde la primavera de 2006 hasta finales de 2008. Se recogieron 109 animales afectados, siendo M. conjunctivae el principal agente causal identificado, $(89,4 \%, \mathrm{n}=104)$, aunque Chlamydophila spp. también fue detectado en un menor porcentaje $(12,5 \%, \mathrm{n}=104)$. Un $4,2 \%(8 / 190)$ de los sarrios cazados y sin lesiones oculares (2006-09) portaban M. conjunctivae, al igual que un 25,8\% (159/617) del ganado ovino-caprino muestreado (17 rebaños, 2008-09). Los ciervos y corzos cazados de las zonas afectadas durante el 2008 fueron negativos a la presencia de $M$. conjunctivae. Un 0,57\% (11/1.924) de los sarrios fue positivo frente a brucelosis siendo negativo el cultivo bacteriológico. La necropsia de 187 sarrios enfermos-muertos mostró un 58,82\% (110) con QCI, un 10,2\% (19) con procesos bronco-neumónicos no parasitarios, un 9,1\% (17) con politraumatismo, un 2,1\% (4) con procesos digestivos, un 1,6\% (3) con otras patologías, y un $18,2 \%$ (34) con procesos indeterminados en animales incompletos. Otras lesiones observadas esporádicamente son dermatofilosis, feocromocitoma, 
hidatidosis y pseudotuberculosis. Los animales cazados $(\mathrm{n}=117)$ presentaban neumonía verminosa, cisticercosis visceral, coccidios y nematodos (Ostertagia spp.) en abomaso. La presencia de áreas con neumonía catarral crónica en lóbulos apicales y Haemonchus spp. en abomaso era frecuente. En los sarrios estudiados no se observaron lesiones sospechosas de tuberculosis ni sarna $(\mathrm{n}=219)$. Ningún sarrio de los analizados $(\mathrm{n}=128)$ en 2008 y 2009 fue seropositivo frente a lengua azul. 


\title{
DINÁMICA DE UN BROTE DE QUERATOCONJUNTIVITIS INFECCIOSA POR MYCOPLASMA CONJUNCTIVAE EN EL SARRIORUPICAPRA P. PYRENAICA
}

\author{
J. Herrero' ${ }^{1}$, C. Prada², O. Fernández-Arberas ${ }^{31}$ \& A. García-Serrano \\ 1. Área de Ecología. Escuela Politécnica Superior de Huesca. Universidad de Zaragoza. E-22071 \\ Zaragoza.herreroj@unizar.es \\ 2. Ega Consultores en Vida Silvestre. Sierra de Vicort 31. E-50003 Zaragoza, Spain. egasl@arrakis.escon \\ 3. Instituto Pirenaico de Ecología. Ap.64. E-22700 Jaca. olatzega@gmail.com
}

Entre 2006 y 2008 tuvo lugar un brote de queratoconjuntivitis infecciosa (QCI) en el sarrio en Aragón y Navarra, así como en el Pirineo francés. El área de estudio fue el Pirineo aragonés y navarro, constituido por 15 macizos montañosos, con un seguimiento poblacional previo, con presencia estable de la especie y en los que 10 de ellos tuvieron casos de QCI. En cinco de ellos hubo además un brote importante acompañado de fuerte mortalidad. Los dos macizos más afectados tuvieron además un impacto en la proporción de sexos en la edad adulta, que favorecía a los machos. El conjunto de la tendencia poblacional entre 2000 y 2007 fue de $\lambda=1.026$, disminuyendo entre 2007 y 2008 a $\lambda=0.8,0.85$ en 2008-2009 y comenzando su recuperación entre 2009 y 2010 con $\lambda=1.1$. El número de sarrios encontrados muertos (2006-10) fue de 470: 31 en 2006, un año sin casi mortalidad debida a la QCI; 220 (2007) y 169 (2008), dos años con fuerte mortalidad por QCI; 34 en 2009 y 16 en 2010, dos años sin mortalidad debida a la enfermedad. Un total de 105 animales enfermos o muertos fueron recogidos y Mycoplasma conjunctivae fue identificado en $93(88.5 \%)$. El brote se extendió en mancha de aceite, hacia el E y el $\mathrm{W}$ desde el punto donde fue detectado por primera vez. Se detectaron dos picos: uno en 2007, que afectó fundamentalmente a un macizo y otro en 2008 que afectó a tres. Los picos fueron estacionales en tres macizos y continuaron desde la primavera hasta el otoño del siguiente año en uno. No encontramos relación entre la densidad, el número y la tendencia con el decrecimiento o el número de animales encontrados muertos, sugiriendo que otros factores juegan un papel en la difusión e impacto de la enfermedad. La caza quedó prácticamente cerrada en los macizos afectados por importante mortalidad, con cupos inferiores al 1\%. Se abrió de nuevo al finalizar el brote y realizar nuevas estimaciones poblacionales. Aunque algunos animales recuperados o no de la enfermedad se encuentran todos los años, este es el primer brote de QCI desde finales de los 70 y principios de los 80 del siglo pasado, hace 30 años, cuando estimas de la guardería indicaron una pérdida del $50 \%$ de la población de las áreas afectadas.

Palabras clave: gestión cinegética, seguimiento poblacional, mortalidad. 
JORNADAS

\title{
DISTANCE SAMPLING EN LA ESTIMA DE ABUNDANCIA DE REBECO
}

\author{
J.M. PéreZ ${ }^{1}$, J.M. López Martín², J. Xifra Corominas ${ }^{3}$, R. Alpizar Jara ${ }^{4}$, \\ I. MARCO ${ }^{5}$, G. MENTABERRE ${ }^{5}$, S. LAVÍ́N ${ }^{5} \&$ E. SERRANO \\ ${ }^{1}$ Departamento de Biología Animal, Biología Vegetal y Ecología, Universidad de Jaén, Campus Las \\ Lagunillas, s.n., 23071, Jaén, Spain. jperez@ujaen.es. \\ 2Direcció General de Medi Natural i Biodiversidad, Departament d' Agricultura, Ramadería, Pesca \\ Alimentació i Medi Natura, E- 08017, Barcelona, España. \\ ${ }^{3}$ Reserva Nacional de Caça Freser-Setcases, Girona, España. \\ ${ }^{4}$ Centro de Investigação em Matemática e Aplicações, Departamento de Matemática, Universidade de \\ Évora, Rua Romão Ramalho, 59, P-7000-671, Évora, Portugal. \\ ${ }^{5}$ Servei d'Ecopatología de la Fauna Salvatge (SEFaS), Facultat de Veterinària, Universitat Autònoma de \\ Barcelona, 08193, Bellaterra, Barcelona, España.
}

La estima de abundancia de una población animal es básica para detectar cambios y tendencias demográficas a diferentes escalas de tiempo. Esta labor es especialmente importante en especies que se someten a aprovechamiento cinegético, ya que los cupos de caza se basan en una estima previa del tamaño de la población. En Europa, las estimas poblacionales de rebeco y de sarrio (Rupicapra spp.) se suelen basar en recuentos totales (censos), que tienden a infraestimar el tamaño total de la población. Además, rara vez se cumplen los supuestos de esta técnica: (i) es imposible muestrear el 100\% del área de estudio, (ii) la probabilidad de observar a todos y cada uno de los individuos no es igual a 1 y (iii) no se puede garantizar que ningún individuo se observa y registra más de una vez.

Durante las últimas décadas, el muestreo por distancias (Distance Sampling - DS) presenta una alternativa a los censos. Esta metodología requiere de la observación de una fracción de la población (muestra) y del registro de la distancia (radial, si se observa desde un punto; o perpendicular a la línea de transecto) al animal o al grupo. A los datos de las distancias observadas son ajustados diferentes modelos probabilísticos que permiten obtener una estima de la densidad y la precisión asociada. A pesar de que esta técnica requiere poco esfuerzo de muestreo, durante la etapa de análisis de los datos hay que tomar varias decisiones que por lo general no están estandarizadas. En este trabajo proponemos un protocolo de obtención de datos y de análisis bajo la metodología DS. Además discutiremos los supuestos del método, la influencia del tamaño de muestra, la importancia del tamaño del área de estudio y la posibilidad de combinar DS con la metodología de captura-marcaje-recaptura, entre otros aspectos.

Palabras clave: censos, Distance Sampling, estima de abundancia, Rupicapra spp. 


\section{Conclusiones}

Cuarenta y ocho participantes del Pirineo y la Cordillera Cantábrica, cazadores, investigadores, guardas, técnicos de las administraciones y consultores, pertenecientes a estos territorios, intercambiaron experiencias y actualizaron su conocimiento multidisciplinar sobre el rebeco cantábrico y el sarrio pirenaico. Algunos de los temas tratados a lo largo de las cuatro sesiones de la jornada han aportado información sobre los siguientes aspectos:

Consideración del sarrio y del rebeco como elementos relevantes de la cultura, historia, paisaje y naturaleza de las montañas ibéricas, valorada y apreciada por sus habitantes y visitantes.

Aportación económica del sarrio a las economías locales de los municipios afectados por las Reservas de Caza.

Incorporación de nuevos actores, tales como grupos conservacionistas, a la conservación de las poblaciones, con experiencias de reintroducción, seguimiento y apoyo a la gestión.

Relevancia de los trabajos de seguimiento a largo plazo y del marcaje poblacional, indispensables para la interpretación de los cambios ocurridos en los diversos parámetros objeto de seguimiento (abundancia, tendencia, tamaño, crecimiento, estado sanitario, etc.).

Existencia de nuevas herramientas de análisis de datos (estadísticas, computacionales, Sistemas de Información Territorial, imágenes satelitales, etc.) y un creciente enfoque multidisciplinar de los fenómenos naturales (ecología, botánica, veterinaria, alimentación, etc.), que deben ser incorporadas a los seguimientos e investigación del sarrio y el rebeco.

Importancia del establecimiento de un seguimiento sanitario adecuado del ganado doméstico estivante en los pastos de montaña, especialmente el ovino, por su relevancia en el mantenimiento y transmisión de enfermedades a la fauna silvestre, particularmente a los ungulados salvajes.

Necesidad de instaurar múltiples indicadores que permitan interpretar los fenómenos naturales que afectan a la evolución de las poblaciones, como los efectos de las cohortes, el cambio global, etc.

Comprobación de cambios de gran magnitud en los ecosistemas de montaña, como son: la matorralización, la disminución de la cabaña ganadera, particularmente de ovino, la composición de la comunidad de ungulados o de la caza menor y la posible modificación de la calidad del pasto.

Destacar la trascendencia de la divulgación del trabajo realizado, tanto a las comunidades locales como a los medios de comunicación, aportando información de calidad e incluyendo el estado de las poblaciones de rebeco y sarrio. 
Relevancia de la existencia de poblaciones no cazadas y cazadas, y de la oportunidad que aportan para la investigación y gestión.

Incomprensión de los cazadores por parte de la sociedad como consecuencia de los cambios socioeconómicos y necesidad de comunicar correctamente los intereses del colectivo y su papel.

Trascendencia de la comprensión del papel que juegan las enfermedades en la dinámica poblacional y urgencia en establecer una red de seguimiento sanitario coordinada.

Considerando las conclusiones y recomendaciones de las Primeras Jornadas pirenaicas sobre el sarrio Rupicapra p. pyrenaica celebradas en Jaca entre el 12 y el 14 de marzo de 2003, se han llevado a cabo cuatro talleres, cuyas principales conclusiones han sido:

Taller sobre el muestreo de distancias (distance sampling). Esta herramienta de estima poblacional ofrece interesantes alternativas a los conteos supraforestales simultáneos tradicionales y su aplicabilidad está siendo estudiada. Un taller en la Reunión sobre Ungulados Silvestres Ibéricos (RUSI) parece una vía adecuada para compartir experiencias sobre su utilización.

Taller sanitario. La normativa europea establece la obligatoriedad de una vigilancia sanitaria (activa y pasiva) de la fauna silvestre. Es necesario reflexionar sobre las enfermedades que deben ser objeto de seguimiento y establecer una formación adecuada de los agentes de campo en lo relativo a la toma de muestras.

Taller sobre cráneos de sarrio. Es necesario mejorar la recogida de datos relativos a la biometría corporal y medición de los cuernos de los animales capturados y cazados, para que aumente el interés de su tratamiento. Esto implica formación y homogeneización en la toma de datos.

Taller sobre gestión poblacional. Algunos temas sobre los que conviene trabajar en los próximos años son: el concepto y desarrollo de la caza selectiva; la mejora de la comercialización de la carne de caza; la actualización del estado de las poblaciones (demográfico y sanitario) unido al intercambio de esta información; la homogeneización de los criterios de gestión entre territorios que comparten poblaciones y la coordinación de los seguimientos poblacionales. 
JORNADAS

\section{Referencias}

Herrero, J., Escudero, E., Fernández De Luco, D. \& García-González, R. (eds.), 2004. El sarrio pirenaico Rupicapra p. pyrenaica: Biología, Patología y Gestión. Consejo de Protección de la Naturaleza de Aragón, vol. 46, 263 pp., Zaragoza.

Pérez-Barbería, F. J. \& Palacios, B. (eds.), 2009. El rebeco cantábrico Rupicapra pyrenaica parva. Conservación y gestión. Organismo Autónomo de Parques Nacionales MMA, 501 pp., Madrid. 